\title{
Relation between $b$-metric and fuzzy metric spaces
}

\author{
Zeinab Hassanzadeh And Shaban Sedghi
}

\begin{abstract}
In this work we have considered several common fixed point results in $b$-metric spaces for weak compatible mappings. By applications of these results we establish some fixed point theorems in $b$-fuzzy metric spaces.
\end{abstract}

\section{INTRODUCTION}

In this paper we establish some fixed point results in a b-fuzzy metric space by applications of certain fixed point theorems in b-metric spaces. Also we prove some fixed point results in b-metric spaces. Fuzzy metric space was first introduced by Kramosil and Michalek [3]. Subsequently, George and Veeramani had given a modified definition of fuzzy metric spaces [1]. Fixed point results in such spaces have been established in a large number of works. Some of these works are noted in $[2,4,5,7,10,11]$.

Definition 1.1. [1] A binary operation $*:[0,1] \times[0,1] \rightarrow[0,1]$ is a continuous t-norm if it satisfies the following conditions:

(1) $*$ is associative and commutative,

(2) $*$ is continuous,

(3) $a * 1=a$, for all $a \in[0,1]$,

(4) $a * b \leq c * d$ whenever $a \leq c$ and $b \leq d$, for each $a, b, c, d \in[0,1]$.

Two typical examples of continuous $t$-norm are $a * b=a b$ and $a * b=$ $\min (a, b)$.

Definition 1.2. [1] A 3-tuple $(X, M, *)$ is called a fuzzy metric space if $X$ is an arbitrary (non-empty) set, $*$ is a continuous $t$-norm and $M$ is a fuzzy set on $X^{2} \times(0, \infty)$, satisfying the following conditions, for each $x, y, z \in X$ and $t, s>0$ :

$$
\text { (1) } M(x, y, t)>0
$$

2010 Mathematics Subject Classification. Primary: 54E40, 54E35, 54H25.

Key words and phrases. Fuzzy contractive mapping, complete fuzzy metric space, common fixed point theorem, weakly compatible maps.

Full paper. Received 2 December 2017, revised 15 March 2018, accepted 7 June 2018, available online 25 June 2018. 
(2) $M(x, y, t)=1$ if and only if $x=y$,

(3) $M(x, y, t)=M(y, x, t)$,

(4) $M(x, y, t) * M(y, z, s) \leq M(x, z, t+s)$,

(5) $M(x, y,):.(0, \infty) \rightarrow[0,1]$ is continuous.

Definition 1.3. [8, 9] A 3-tuple $(X, M, *)$ is called a $b$-fuzzy metric space if $X$ is an arbitrary (non-empty) set, $*$ is a continuous $t$-norm and $M$ is a fuzzy set on $X^{2} \times(0, \infty)$, satisfying the following conditions, for each $x, y, z \in X$, $t, s>0$ and a given real number $b \geq 1$ :

(1) $M(x, y, t)>0$,

(2) $M(x, y, t)=1$ if and only if $x=y$,

(3) $M(x, y, t)=M(y, x, t)$,

(4) $M\left(x, y, \frac{t}{b}\right) * M\left(y, z, \frac{s}{b}\right) \leq M(x, z, t+s)$,

(5) $M(x, y,):.(0, \infty) \rightarrow[0,1]$ is continuous.

We present an example shows that a $b$-fuzzy metric on $X$ need not be a fuzzy metric on $\mathrm{X}$.

Example 1.4. Let $M(x, y, t)=e^{\frac{-|x-y|^{p}}{t}}$, where $p>1$ is a real number. We show that $M$ is a $b$-fuzzy metric with $b=2^{p-1}$.

Obviously conditions (1), (2), (3) and (5) of Definition 1.3 are satisfied.

If $1<p<\infty$, then the convexity of the function $f(x)=x^{p}(x>0)$ implies

$$
\left(\frac{a+c}{2}\right)^{p} \leq \frac{1}{2}\left(a^{p}+c^{p}\right)
$$

and hence, $(a+c)^{p} \leq 2^{p-1}\left(a^{p}+c^{p}\right)$ holds. Therefore,

$$
\begin{aligned}
\frac{|x-y|^{p}}{t+s} & \leq 2^{p-1} \frac{|x-z|^{p}}{t+s}+2^{p-1} \frac{|z-y|^{p}}{t+s} \\
& \leq 2^{p-1} \frac{|x-z|^{p}}{t}+2^{p-1} \frac{|z-y|^{p}}{s} \\
& =\frac{|x-z|^{p}}{t / 2^{p-1}}+\frac{|z-y|^{p}}{s / 2^{p-1}} .
\end{aligned}
$$

Thus for each $x, y, z \in X$ we obtain

$$
\begin{aligned}
M(x, y, t+s) & =e^{\frac{-|x-y|^{p}}{t+s}} \\
& \geq M\left(x, z, \frac{t}{2^{p-1}}\right) * M\left(z, y, \frac{s}{2^{p-1}}\right),
\end{aligned}
$$

where $a * b=a b$. So condition (4) of Definition 1.3 hold and $M$ is a $b$-fuzzy metric.

It should be noted that in preceding example, for $p=2$ it is easy to see that $(X, M, *)$ is not a fuzzy metric space. 
Example 1.5. Let $M(x, y, t)=e^{\frac{-d(x, y)}{t}}$ or $M(x, y, t)=\frac{t}{t+d(x, y)}$, where $d$ is a $b$-metric on $\mathrm{X}$ and $a * c=a c$, for all $a, c \in[0,1]$. Then it is easy to show that $M$ is a $b$-fuzzy metric.

Obviously conditions (1), (2), (3) and (5) of Definition 1.3 are satisfied. For each $x, y, z \in X$ we obtain

$$
\begin{aligned}
M(x, y, t+s) & =e^{\frac{-d(x, y)}{t+s}} \\
& \geq e^{-b \frac{d(x, z)+d(z, y)}{t+s}} \\
& =e^{-b \frac{d(x, z)}{t+s}} \cdot e^{-b \frac{d(z, y)}{t+s}} \\
& \geq e^{\frac{-d(x, z)}{t / b}} \cdot e^{\frac{-d(z, y)}{s / b}} \\
& =M\left(x, z, \frac{t}{b}\right) * M\left(z, y, \frac{s}{b}\right) .
\end{aligned}
$$

So condition (4) of Definition 1.3 is hold and $M$ is a $b$-fuzzy metric. Similarly, it is easy to see that $M(x, y, t)=\frac{t}{t+d(x, y)}$ is a $b$-fuzzy metric.

\section{MAIN RESULTS}

Lemma 2.1. Let $(X, M, *)$ be a b-fuzzy metric space with $a * c \geq a c$, for all $a, c \in[0,1]$. If $d: X^{2} \rightarrow[0, \infty)$ is defined by $d(x, y)=\lim _{\epsilon \rightarrow 0} \int_{\epsilon}^{1} \log _{\alpha} M(x, y, t) d t$, for $0<\alpha<1$, then $d$ is an 2b-metric on $X$.

Proof. By definition, we have that $d(x, y)$ is well defined for each $x, y \in X$. Clearly, $d(x, y) \geq 0$, for all $x, y \in X$. Moreover, $d(x, y)=0$ if and only if $\log _{\alpha}(M(x, y, t))=0$ if and only if $M(x, y, t)=1$ if and only if $x=y$.

Since

$$
\begin{aligned}
M(x, y, t) & \geq M\left(x, z, \frac{t}{2 b}\right) * M\left(z, y, \frac{t}{2 b}\right) \\
& \geq M\left(x, z, \frac{t}{2 b}\right) \cdot M\left(z, y, \frac{t}{2 b}\right),
\end{aligned}
$$

it follows that

$$
\begin{aligned}
d(x, y) & =\lim _{\epsilon \rightarrow 0} \int_{\epsilon}^{1} \log _{\alpha} M(x, y, t) d t \\
& \leq \lim _{\epsilon \rightarrow 0} \int_{\epsilon}^{1} \log _{\alpha} M\left(x, z, \frac{t}{2 b}\right) \cdot M\left(z, y, \frac{t}{2 b}\right) d t \\
& \leq \lim _{\epsilon \rightarrow 0} \int_{\epsilon}^{1} \log _{\alpha} M\left(x, z, \frac{t}{2 b}\right) d t+\lim _{\epsilon \rightarrow 0} \int_{\epsilon}^{1} \log _{\alpha} M\left(z, y, \frac{t}{2 b}\right) d t \\
& =2 b \lim _{\epsilon \rightarrow 0} \int_{\frac{\epsilon}{2 b}}^{\frac{1}{2 b}} \log _{\alpha} M(x, z, t) d t+2 b \lim _{\epsilon \rightarrow 0} \int_{\frac{\epsilon}{2 b}}^{\frac{1}{2 b}} \log _{\alpha} M(z, y, t) d t \\
& \leq 2 b\left[\lim _{\epsilon \rightarrow 0} \int_{\frac{\epsilon}{2 b}}^{1} \log _{\alpha} M(x, z, t) d t+\lim _{\epsilon \rightarrow 0} \int_{\frac{\epsilon}{2 b}}^{1} \log _{\alpha} M(x, z, t) d t\right]
\end{aligned}
$$




$$
=2 b[d(x, z)+d(z, y)] .
$$

This proves that $d$ is an $2 b$-metric on $\mathrm{X}$.

The following lemma plays an important role to give fixed point results on a fuzzy metric space.

Lemma 2.2. Let $(X, M, *)$ be a b-fuzzy metric space with $a * c \geq a c$, for all $a, c \in[0,1]$. If $d: X^{2} \rightarrow[0, \infty)$ is define by $d(x, y)=\lim _{\epsilon \rightarrow 0} \int_{\epsilon}^{1} \log _{\alpha} M(x, y, t) d t$, for all $0<\alpha<1$, then:

(1) $\left\{x_{n}\right\}$ is a Cauchy sequence in b-fuzzy metric $(X, M, *)$ if and only if it is a Cauchy sequence in the $2 b$ - metric space $(X, d)$.

(2) A b-fuzzy metric space $(X, M, *)$ is complete if and only if the $2 b$ metric space $(X, d)$ is complete.

Proof. First we show that every Cauchy sequence in $(X, M, *)$ is a Cauchy sequence in $(X, d)$. To this end let $\left\{x_{n}\right\}$ be a Cauchy sequence in $(X, M, *)$. Then $\lim _{n, m \rightarrow \infty} M\left(x_{n}, x_{m}, t\right)=1$. Since

$$
d\left(x_{n}, x_{m}\right)=\lim _{\epsilon \rightarrow 0} \int_{\epsilon}^{1} \log _{\alpha} M\left(x_{n}, x_{m}, t\right) d t
$$

is a $2 b$-metric. Hence, we have

$$
\begin{aligned}
\lim _{n, m \rightarrow \infty} d\left(x_{n}, x_{m}\right) & =\lim _{n, m \rightarrow \infty} \lim _{\epsilon \rightarrow 0} \int_{\epsilon}^{1} \log _{\alpha} M\left(x_{n}, x_{m}, t\right) d t \\
& =\lim _{\epsilon \rightarrow 0} \int_{\epsilon}^{1} \log _{\alpha} \lim _{n, m \rightarrow \infty} M\left(x_{n}, x_{m}, t\right) d t=0,
\end{aligned}
$$

so, we conclude that $\left\{x_{n}\right\}$ is a Cauchy sequence in $(X, d)$.

Next we prove that completeness of $(X, d)$ implies completeness of $(X, M, *)$. Indeed, if $\left\{x_{n}\right\}$ is a Cauchy sequence in $(X, M, *)$ then it is also a Cauchy sequence in $(X, d)$. Since the $2 b$-metric space $(X, d)$ is complete we deduce that there exists $y \in X$ such that $\lim _{n \rightarrow \infty} d\left(x_{n}, y\right)=0$. Therefore,

$$
\lim _{n \rightarrow \infty \epsilon \rightarrow 0} \int_{\epsilon}^{1} \log _{\alpha} M\left(x_{n}, y, t\right) d t=\lim _{\epsilon \rightarrow 0} \int_{\epsilon}^{1} \log _{\alpha} \lim _{n \rightarrow \infty} M\left(x_{n}, y, t\right) d t=0,
$$

that is $\lim _{n \rightarrow \infty} M\left(x_{n}, y, t\right) d t=1$. Hence we follow that $\left\{x_{n}\right\}$ is a convergent sequence in $(X, M, *)$.

Now we prove that every Cauchy sequence $\left\{x_{n}\right\}$ in $(X, d)$ is a Cauchy sequence in $(X, M, *)$. Since $\left\{x_{n}\right\}$ is a Cauchy sequence in $(X, d)$, then

$$
\begin{aligned}
\lim _{n, m \rightarrow \infty} d\left(x_{n}, x_{m}\right) & =\lim _{n, m \rightarrow \infty \epsilon \rightarrow 0} \int_{\epsilon}^{1} \log _{\alpha} M\left(x_{n}, x_{m}, t\right) d t \\
& =\lim _{\epsilon \rightarrow 0} \int_{\epsilon}^{1} \log _{\alpha} \lim _{n, m \rightarrow \infty} M\left(x_{n}, x_{m}, t\right) d t=0 .
\end{aligned}
$$


Hence, $\lim _{n, m \rightarrow \infty} M\left(x_{n}, x_{m}, t\right)=1$.

That is, $\left\{x_{n}\right\}$ is a Cauchy sequence in $(X, M, *)$.

We will establish the lemma if we prove that $(X, d)$ is complete if so is $(X, M, *)$. Let $\left\{x_{n}\right\}$ be a Cauchy sequence in $(X, d)$. Then $\left\{x_{n}\right\}$ is a Cauchy sequence in $(X, M, *)$, and so it is convergent to a point $y \in X$ with

$$
\lim _{n \rightarrow \infty} M\left(x_{n}, y, t\right)=1 \text {. }
$$

As a consequence we have

$$
\begin{aligned}
\lim _{n \rightarrow \infty} d\left(x_{n}, y\right) & =\lim _{n \rightarrow \infty} \lim _{\epsilon \rightarrow 0} \int_{\epsilon}^{1} \log _{\alpha} M\left(x_{n}, y, t\right) d t \\
& =\lim _{\epsilon \rightarrow 0} \int_{\epsilon}^{1} \log _{\alpha} \lim _{n \rightarrow \infty} M\left(x_{n}, y, t\right) d t=0 .
\end{aligned}
$$

Therefore $(X, d)$ is complete.

Lemma 2.3. Let $(X, M, *)$ be a b-fuzzy metric space with $a * c=\min \{a, c\}$, for all $a, c \in[0,1]$. We define $d: X^{2} \rightarrow[0, \infty)$ by

$$
d(x, y)=\lim _{\epsilon \rightarrow 0} \int_{\epsilon}^{1} \cot \left(\frac{\pi}{2} M(x, y, t)\right) d t,
$$

then $d$ is an 2b-metric on $X$.

Proof. Clearly, $d(x, y) \geq 0$, for all $x, y \in X$. Moreover, $d(x, y)=0$ if and only if $\cot \left(\frac{\pi}{2} M(x, y, t)\right)=0$ if and only if $M(x, y, t)=1$ if and only if $x=y$.

Since,

$$
M(x, y, t) \geq M\left(x, z, \frac{t}{2 b}\right) * M\left(z, y, \frac{t}{2 b}\right)=\min \left\{M\left(x, z, \frac{t}{2 b}\right), M\left(z, y, \frac{t}{2 b}\right)\right\},
$$

and also since $0<\frac{\pi}{2} M\left(x, y, \frac{t}{2 b}\right) \leq \frac{\pi}{2}$, it follows that,

$$
\begin{aligned}
d(x, y) & =\lim _{\epsilon \rightarrow 0} \int_{\epsilon}^{1} \cot \left(\frac{\pi}{2} M(x, y, t)\right) d t \\
& \leq \lim _{\epsilon \rightarrow 0} \int_{\epsilon}^{1} \cot \left[\frac{\pi}{2}\left(M\left(x, z, \frac{t}{2 b}\right) * M\left(z, y, \frac{t}{2 b}\right)\right)\right] d t \\
& =2 b\left(\lim _{\epsilon \rightarrow 0} \int_{\frac{\epsilon}{2 b}}^{\frac{1}{2 b}} \cot \left(\frac{\pi}{2} \min \{M(x, z, t), M(z, y, t)\}\right) d t\right) \\
& =2 b \min _{\frac{\epsilon}{\epsilon \rightarrow 0}}\left\{\int_{\frac{\epsilon}{2 b}}^{\frac{1}{2 b}} \cot \left(\frac{\pi}{2} M(x, z, t)\right) d t, \lim _{\epsilon \rightarrow 0} \int_{\frac{\epsilon}{2 b}}^{\frac{1}{2 b}} \cot \left(\frac{\pi}{2} M(z, y, t)\right) d t\right\} \\
& \leq 2 b \lim _{\epsilon \rightarrow 0} \int_{\frac{\epsilon}{2 b}}^{1} \cot \left(\frac{\pi}{2} M(x, z, t)\right) d t+2 b \lim _{\epsilon \rightarrow 0} \int_{\frac{\epsilon}{2 b}}^{1} \cot \left(\frac{\pi}{2} M(z, y, t)\right) d t \\
& =2 b[d(x, z)+d(z, y)],
\end{aligned}
$$

that is $d$ is an $2 b$-metric on $X$. 
Remark 2.4. Let $a, b \in(0,1]$, then it is a standard result that

$$
\operatorname{arccot}(\min \{a, b\}) \leq \operatorname{arccot}(a)+\operatorname{arccot}(b)-\frac{\pi}{4} .
$$

Lemma 2.5. Let $(X, M, *)$ be a $2 b$-fuzzy metric space with $a * c=\min \{a, c\}$, for all $a, c \in[0,1]$. If we define $d: X^{2} \rightarrow[0, \infty)$ by

$$
d(x, y)=\lim _{\epsilon \rightarrow 0} \int_{\epsilon}^{1}\left(\frac{4}{\pi} \operatorname{arccot}(M(x, y, t))-1\right) d t,
$$

then $d$ is an $2 b$-metric on $X$.

Proof. Clearly, $0 \leq d(x, y)<1$, for all $x, y \in X$. Moreover, $d(x, y)=0$ if and only if $\frac{4}{\pi} \operatorname{arccot}(M(x, y, t))-1=0$ if and only if $\operatorname{arccot}(M(x, y, t))=\frac{\pi}{4}$ if and only if $M(x, y, t)=1$ if and only if $x=y$. Since

$$
M(x, y, t) \geq M\left(x, z, \frac{t}{2 b}\right) * M\left(z, y, \frac{t}{2 b}\right)=\min \left\{M\left(x, z, \frac{t}{2 b}\right), M\left(z, y, \frac{t}{2 b}\right)\right\},
$$

it follows that

$$
\begin{aligned}
\operatorname{arccot}(M(x, y, t)) & \leq \operatorname{arccot}\left[M\left(x, z, \frac{t}{2 b}\right) * M\left(z, y, \frac{t}{2 b}\right)\right] \\
& =\operatorname{arccot}\left(\min \left\{M\left(x, z, \frac{t}{2 b}\right), M\left(z, y, \frac{t}{2 b}\right)\right\}\right) \\
& \leq \operatorname{arccot}\left(M\left(x, z, \frac{t}{2 b}\right)\right)+\operatorname{arccot}\left(M\left(z, y, \frac{t}{2 b}\right)\right)-\frac{\pi}{2} .
\end{aligned}
$$

Hence,

$$
\begin{aligned}
& d(x, y)= \lim _{\epsilon \rightarrow 0} \int_{\epsilon}^{1}\left(\frac{4}{\pi} \operatorname{arccot}(M(x, y, t))-1\right) d t \\
& \leq \lim _{\epsilon \rightarrow 0} \int_{\epsilon}^{1}\left(\frac{4}{\pi} \operatorname{arccot}\left(M\left(x, z, \frac{t}{2 b}\right)\right)-1\right) d t \\
& \quad+\lim _{\epsilon \rightarrow 0} \int_{\epsilon}^{1}\left(\frac{4}{\pi} \operatorname{arccot}\left(M\left(z, y, \frac{t}{2 b}\right)\right)-1\right) d t \\
&=2 b \lim _{\epsilon \rightarrow 0} \int_{\frac{\epsilon}{2 b}}^{\frac{1}{2 b}}\left(\frac{4}{\pi} \operatorname{arccot}(M(x, z, t))-1\right) d t \quad+2 b \lim _{\epsilon \rightarrow 0} \int_{\frac{\epsilon}{2 b}}^{\frac{1}{2 b}}\left(\frac{4}{\pi} \operatorname{arccot}(M(z, y, t))-1\right) d t \\
& \leq 2 b\left(\lim _{\epsilon \rightarrow 0} \int_{\frac{\epsilon}{2 b}}^{1}\left(\frac{4}{\pi} \operatorname{arccot}(M(x, z, t))-1\right) d t\right. \\
&\left.\quad+\lim _{\epsilon \rightarrow 0} \int_{\frac{\epsilon}{2 b}}^{1}\left(\frac{4}{\pi} \operatorname{arccot}(M(z, y, t))-1\right) d t\right) \\
&=2 b[d(x, z)+d(z, y)],
\end{aligned}
$$

that is $d$ is an $2 b$-metric on $X$. 
Remark 2.6. Let $(X, M, *)$ be a fuzzy metric space with $a * c \geq a c$, for all $a, c \in[0,1]$. If sequence $\left\{x_{n}\right\}$ in $X$ converges to $x$, that is, for every $0<\epsilon<1$ there exists $n_{0} \in \mathbb{N}$ such that $M\left(x_{n}, x, t\right)>1-\epsilon$, for all $n \geq n_{0}$ and each $t>0$, then $d\left(x_{n}, x\right) \rightarrow 0$ where $d(x, y)=\lim _{\epsilon \rightarrow 0} \int_{\epsilon}^{1} \log _{\alpha} M(x, y, t) d t$. Also it is a Cauchy sequence if for each $0<\epsilon<1$ and $t>0$, there exits $n_{0} \in \mathbb{N}$ such that $M\left(x_{n}, x_{m}, t\right)>1-\epsilon$ for each $n, m \geq n_{0}$. It follows that $d\left(x_{n}, x_{m}\right)=\lim _{\epsilon \rightarrow 0} \int_{\epsilon}^{1} \log _{\alpha} M\left(x_{n}, x_{m}, t\right) d t<\lim _{\epsilon \rightarrow 0} \int_{\epsilon}^{1} \log _{\alpha}(1-\epsilon) d t<\eta$, for every $\eta=(1-\alpha) \log _{\alpha}(1-\epsilon)$. Thus $\left\{x_{n}\right\}$ in $2 b$-metric $(X, d)$ is a Cauchy sequence.

Theorem 2.7. [6] Suppose that $f, g, S$ and $T$ are self mappings of a complete b-metric space $(X, d)$, with $f(X) \subseteq T(X), g(X) \subseteq S(X)$ and that the pairs $\{f, S\}$ and $\{g, T\}$ are compatible. If

$d(f x, g y) \leq \frac{q}{b^{4}} \max \left\{d(S x, T y), d(f x, S x), d(g y, T y), \frac{1}{2}(d(S x, g y)+d(f x, T y))\right\}$,

for each $x, y \in X$, with $0<q<1$. Then $f, g, S$ and $T$ have a unique common fixed point in $X$ provided that $S$ and $T$ are continuous.

We next apply theorem 2.7 to establish the following theorem in fuzzy metric spaces.

Theorem 2.8. Let $(X, M, *)$ be a complete fuzzy metric space with $a * c \geq a c$ for all $a, c \in[0,1]$. Let $f, g, S$ and $T$ be self mappings on $X$ with $f(X) \subseteq$ $T(X), g(X) \subseteq S(X)$ and that the pairs $\{f, S\}$ and $\{g, T\}$ are compatible. If there exists $q \in(0,1)$ such that for each $x, y \in X$,

$M(f x, g y, t) \geq \min \left(\begin{array}{l}M(S x, T y, t), M(f x, S x, t), \\ M(g y, T y, t), \sqrt{M(S x, g y, t) \cdot M(f x, T y, t))}\end{array}\right)^{\frac{q}{(2 b)^{4}}}$

If $S$ and $T$ are continuous, then $f, g, S$ and $T$ have a unique common fixed point in $X$.

Proof. We define $d(x, y)=\lim _{\epsilon \rightarrow 0} \int_{\epsilon}^{1} \log _{\alpha} M(x, y, t) d t$ for every $x, y \in X$ where $0<\alpha<1$. Then by Lemma 2.1 and Lemma $2.2(X, d)$ is a complete $2 b$-metric space. From the above inequality, we get, 


$$
\begin{aligned}
& \lim _{\epsilon \rightarrow 0} \int_{\epsilon}^{1} \log _{\alpha} M(f x, g y, t) d t \leq \\
& \quad \frac{q}{(2 b)^{4}} \max \left(\begin{array}{l}
\lim _{\epsilon \rightarrow 0} \int_{\epsilon}^{1} \log _{\alpha} M(S x, T y, t) d t, \lim _{\epsilon \rightarrow 0} \int_{\epsilon}^{1} \log _{\alpha} M(f x, S x, t) d t, \\
\lim _{\epsilon \rightarrow 0} \int_{\epsilon}^{1} \log _{\alpha} M(g y, T y, t) d t, \\
\frac{1}{2}\left(\lim _{\epsilon \rightarrow 0} \int_{\epsilon}^{1} \log _{\alpha} M(S x, g y, t) d t+\lim _{\epsilon \rightarrow 0} \int_{\epsilon}^{1} \log _{\alpha} M(f x, T y, t) d t\right)
\end{array}\right),
\end{aligned}
$$

which is,

$$
d(f x, g y) \leq \frac{q}{(2 b)^{4}} \max \left(\begin{array}{l}
d(S x, T y), d(f x, S x) \\
d(g y, T y), \frac{1}{2}(d(S x, g y)+d(f x, T y))
\end{array}\right)
$$

Hence all the conditions of Theorem 2.7 hold, so the conclusion of Theorem 2.8 follows by an application of Theorem 2.7.

\section{REFERENCES}

[1] A. George, P. Veeramani, On some result in fuzzy metric space, Fuzzy Sets Systems, 64 (1994), 395-399.

[2] V. Gregori, A. Sapena, On fixed-point theorem in fuzzy metric spaces, Fuzzy Sets and Systems, 125 (2002), 245-252.

[3] I. Kramosil, J. Michalek, Fuzzy metric and statistical metric spaces, Kybernetica 11 (1975), 326-34.

[4] J. Rodríguez López, S. Ramaguera, The Hausdorff fuzzy metric on compact sets, Fuzzy Sets and Systems, 147 (2004), 273-283.

[5] D. Mihet, A Banach contraction theorem in fuzzy metric spaces, Fuzzy Sets and Systems, 144 (2004), 431-439.

[6] J. R. Roshan, N. Shobkolaei, S. Sedghi, M. Abbas, Common fixed point of four maps in b-metric spaces, Hacettepe Journal of Mathematics and Statistics, 43 (4) (2014), 613-624.

[7] S. Sedghi, B. S. Choudhury, N. Shobe, Unique common fixed point theorem for four weakly compatible mappings in complete fuzzy metric spaces, The Journal of Fuzzy Mathematics, 18 (1) (2010) 161-170.

[8] S. Sedghi, N. Shobe, Common fixed point Theorem in b-fuzzy metric space, Nonlinear Functional Analysis and Applications, 17 (3) (2012), 349-359.

[9] S. Sedghi, N. Shobe, Common fixed point Theorem for R-Weakly Commuting Maps in b-fuzzy Metric Space, Nonlinear Functional Analysis and Applications, 19 (2) (2014), 285-295. 
[10] R. Vasuki, P. Veeramani, Fixed point theorems and Cauchy sequences in fuzzy metric spaces, Fuzzy Sets and Systems, 135 (2003), 409-413.

[11] D. Xieping, Common fixed point theorem of commuting mappings in PM-spaces, Kexue Tongbao, 29 (1984), 147-150.

\section{Zeinab Hassanzadeh}

Department of Mathematics

QAEMSHAHR BRANCH

ISLAMIC AZAD UNIVERSITY

QAEMSHAHR

IRAN

E-mail address: Z.hassanzadeh1368@yahoo.com

\section{Shaban Sedghi}

Department of Mathematics

QAEMSHAHR BRANCH

ISLAMIC AZAD UNIVERSITY

QAEMSHAHR

IRAN

E-mail address: sedghi_gh@yahoo.com

sedghi.gh@qaemiau.ac.ir 